\title{
Quelques données sur la population de Proasellus slavus ssp. $n$. Sket (Crustacea, Isopoda) dans l'hyporhéique de la rivière Drave près de Legrad
}

\author{
par \\ Romana LATTINGER-PENKO*
}

\begin{abstract}
SUMMARY
New data on the population of Proasellus slavus ssp. $n$. Sket

(Crustacea, Isopoda) in the hyporheic water of the Drava river near Legrad
\end{abstract}

\begin{abstract}
An analysis of the population in the hyporheic medium of the Drava river shows that Proasellus slavus ssp. $\mathrm{n}$. is one of the smallest known Asellids. It is living in an interstitial biotope as a permanent population in which density varies in space and time. Our observations show that reproduction takes place throughout the year, however with a maximum rate at the beginning of summer. Females may produce a relatively great number of offspring, notwithstanding the smallness of the species.
\end{abstract}

\section{INTRODUCTION}

Lors de recherches sur la pollution des eaux de la Drave et sur son influence sur la faune des eaux hypogées voisines, un assez grand nombre d'exemplaires de Crustacés Isopodes anophtalmes et dépigmentés, appartenant à une sousespèce de Proasellus slavus, ont été récoltés.

Cette forme a été décrite pour la première fois sous le nom d'Asellus slavus ssp. sur la base de trois femelles provenant d'un puits creusé à Gornja Radgona le long de la rivière Mura (Sket, 1963). Nos récoltes, elles, ont été faites dans le milieu hyporhéique. Quelques unes des particularités physiques et chimiques de ce biotope, la répartition, l'inventaire et la densité de la population ainsi que la composition de la biocénose où elle vit, sont décrites dans un travail en commun (Mestrov et al., 1976).

Les observations présentées ici complètent ce travail en fournissant non seulement les données numériques complètes sur la composition de la population, la sex-ratio et les catégories de taille mais aussi le rapport existant entre l'activité de reproduction et certaines conditions du milieu.

* Zoologijski zavod PMF, Zagreb I, Rooseveltov TRG 6/1, Yougoslavie. 


\section{MATERIEL ET METHODES}

Les animaux sont prélevés par les méthodes de Bou-Rouch et Karaman-Chappuis sur la rive droite de la rivière Drave dans un lit de gravier et de sable et dans un banc voisin de même structure. Le lieu d'étude est situé à quelques kilomètres au Sud-Est du village de Legrad, près du confluent de la Mura et de la Drave.

Le matériel est récolté à une profondeur allant jusqu'à $2 \mathrm{~m}$ et on est allé jusqu'à $6 \mathrm{~m}$ depuis le bord de la rivière vers son milieu et à $5 \mathrm{~m}$ sur le rivage. Tous les sondages ont été effectués dans le cadre d'une superficie d'environ $100 \mathrm{~m}^{2}$.

Les prélèvements effectués ont été répartis sur un an et 8 mois et ont été limités à cinq (les 12 juillet 1973, 3 septembre 1974, 21 avril 1974, 28 juillet 1974 et 27 mars 1975) en raison de l'inaccessibilité du lieu pendant les crues.

Il fallait habituellement pomper jusqu'à ce que les eaux s'arrêtent de couler, et si l'eau était abondante, ce qui était rare, on s'arrêtait après 40-100 litres d'eau filtrée.

Outre la température, on mesurait quelques particularités chimiques de l'eau (quantité d'oxygène dissous, déficit d'oxygène, quantité de $\mathrm{CO}_{2}$ dissous, alcalinité, dureté de l'eau et pH), puis degré de saprobité de l'eau (Meštrov et al., 1976).

Ont été pris au total 1253 exemplaires de Proasellus slavus ssp. n.. La longueur du corps, le sexe et le degré de développement (larves plus jeunes ou plus âgées, adultes) de tous ceux qui ont été suffisamment bien conçerves ont été déterminés.

Les phases de développement n'ont pas été étudiées, et dans ce travail n'a été utilisée que la division provisoire en larves plus ou moins jeunes.

Les animaux abimés par le mode de prélèvement n'ont pas fait l'objet de mesures de la longueur du corps, mais ils servaient pour le dénombrement et pour l'analyse de la population par sexe en se basant sur les parties postérieures de corps possédant des pléopodes differenciés.

Les mesures ont été prises au moyen d'un stéréomicroscope, et la longueur du corps est évaluée de la base des antennes à l'extrémité du pléotelson.

\section{RESULTATS}

\section{Sex-ratio}

On peut dire que dans la population de Proasellus slavus ssp. n. près de Legrad, les mâles prédominent puisque seulement dans 5 sur 21 échantillons les femelles sont en majorité. $\mathrm{Si}$, par contre, nous faisons la somme des exemplaires pris à la même date, on constate que les femelles sont plus nombreuses dans seulement un échantillon (mars). Dans tous les autres échantillons, il y a de 1,3 à 3,4 fois plus de mâles que de femelles. Dans la récolte totale, le rapport des mâles et des femelles adultes est de 1,6:1.

Sur le nombre total des animaux récoltés (1253) les mâles représentent 
$39,74 \%$ (498 exemplaires), et les femelles $25,13 \%$ (315 exemplaires dont 39 , soit $3,11 \%$ du total sont pourvues d'oostégites). Le reste de la population est formée par des phases de développement et par des animaux dont le sexe n'est pas déterminable (fig. 1).

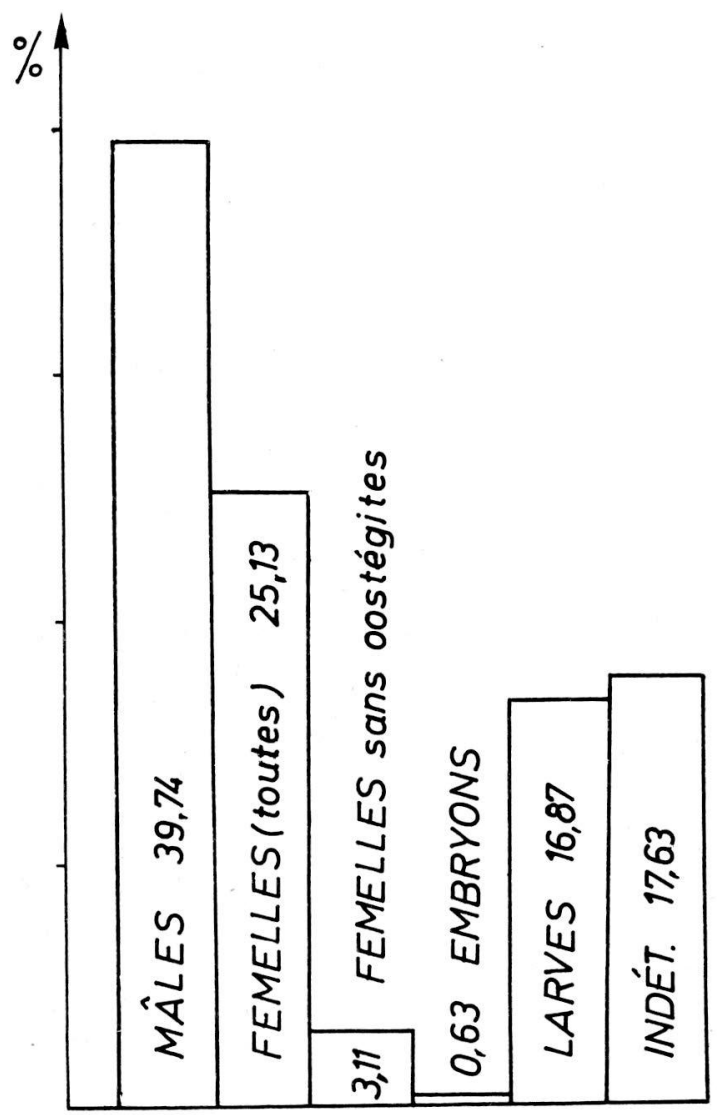

Fig. 1. Proascllus slavus ssp. n. Structure de la population en pourcentage.

Composition de la population d'après la taille (longueur du corps).

Parmi tous les exemplaires, il n'y en avait que 704 pour lesquels il a été possible de déterminer le sexe et la longueur du corps. Le reste était constitué de larves et d'adultes au corps endommagé dont les caractéristiques ne pouvaient être définies.

La longueur du corps des mâles est de 1,5 à $5 \mathrm{~mm}$, et celle des femelles de 1,5 à $4,2 \mathrm{~mm}$, mais la taille dominante des mâles est de $3 \mathrm{~mm}$, celle des femelles 
$2 \mathrm{~mm}$. Les dimensions minimales des animaux adultes concordent avec la taille à laquelle on peut à peine différencier morphologiquement les mâles des femelles, soit 1,5 mm. Chez les plus jeunes, il semble que les pléopodes sexuels ne soient pas encore différenciés. Ces petits exemplaires sont encore à l'état de larve. Les femelles trouvées avec oostégites plus ou moins développés n'avaient pas moins de $2,5 \mathrm{~mm}$. Leur longueur est de l'ordre de 2,5 à $4 \mathrm{~mm}$. Chez la plupart le marsupium était fonctionnel: 4 paires de grands oostégites ovales et une paire de petits et ronds du maxillipède avec longues soies ferment l'espace ovale du marsupium. Ce dernier s'étend ainsi depuis la tête jusqu'au bord postérieur du cinquième segment thoracique.

Presque tous les marsupiums étaient béants par suite d'un écartement des oostégites, conséquence de la brutalité de la méthode de capture, aussi n'est-il pas étonnant que nous n'ayons trouvé d'embryons ou de larves que dans quelques rares femelles, et ce partiellement: dans l'échantillon du 12 juillet 1973 prélevé à $1 \mathrm{~m}$ de profondeur du fond de la rivière, il y avait une femelle de 3,2 mm de longueur avec 9 larves de $0,8 \mathrm{~mm}$; dans l'échantillon pris à $1,5 \mathrm{~m}$ de profondeur, le banc de sable a fourni une femelle de $3 \mathrm{~mm}$ avec 3 oeufs et une autre femelle de $3 \mathrm{~mm}$ avec 15 oeufs embryonnés dans un marsupium bien fermé qui, cependant, ne semblait pas aussi rempli qu'il aurait pu l'être.

Des femelles avec oostégites non-fonctionnels ont parfois été rencontrées: le 12 juillet 1974 une de 3 et une autre de $3,2 \mathrm{~mm}$ parmi 13 pourvues de marsupium fonctionnel; le 17 mars 1975 cinq mais aucune femelle à marsupium.

Huit embryons trouvés dans le filtrat avaient la grosseur et la forme des jeunes larves, enfermés dans la cuticule d'un oeuf allongé, ovale et transparent. Ils ont été capturés le 12 juillet 1973 dans le fond de la rivière, à une profondeur de $0,5 \mathrm{~m}$ et à $6 \mathrm{~m}$ du rivage ( 3 embryons), puis à une profondeur de $2 \mathrm{~m}$, à $1 \mathrm{~m} \mathrm{du}$ rivage ( 2 embryons).

Le même jour on a également récolté deux exemplaires dans le banc de sable, à $1 \mathrm{~m}$ de profondeur et à $1,5 \mathrm{~m}$ du bord de la rivière et le 17 mars 1975 encore un embryon, et ce, au bord de la rivière, à $0,7 \mathrm{~m}$ de profondeur.

Les plus petites larves capturées, celles de $0,8 \mathrm{~mm}$ de longueur, ont encore le corps courbé en forme d'arc, avec les bourgeons des extrémités de formes égales se présentant comme de petits rouleaux sans segments s'avancant en dehors, par couples, sur le côté ventral du corps. Dans leur développement ultérieur, les bourgeons des péréiopodes s'allongent, se segmentent et pendent du côté ventral. Les articles sont toujours en forme de rouleaux et n'ont pas de formes caractéristiques. La longueur du corps varie de 1 à $1,5 \mathrm{~mm}$. Les organes dorsaux existent encore. 65 larves de ce genre ont été trouvées; ne connaissant pas le nombre de phases larvaires, nous les désignons par le terme "larves plus jeunes".

Les formes de 1 à $1,8 \mathrm{~mm}$ et, exceptionnellement jusqu'à $2 \mathrm{~mm}$ ont été appelées "larves plus âgées"; elles ont toutes les péréiopodes parfaitement développés, mais leurs pléopodes sexuels ne sont pas encore différenciés, ou tout au moins ne peuvent être discernés sans dissection. 147 exemplaires de ce type ont été trouvés.

Parmi les animaux de cette dimension on compte une centaine d'exemplaires 
qui, à cause de l'endommagement du corps, en général à cause des pattes arrachées, n'ont pu être compris avec certitude dans aucun groupe dans la composition de la population. Dans la Fig. 1, ils sont inclus parmi les exemplaires non définis, mais d'après la longueur du corps, on peut supposer qu'il s'agit de larves.
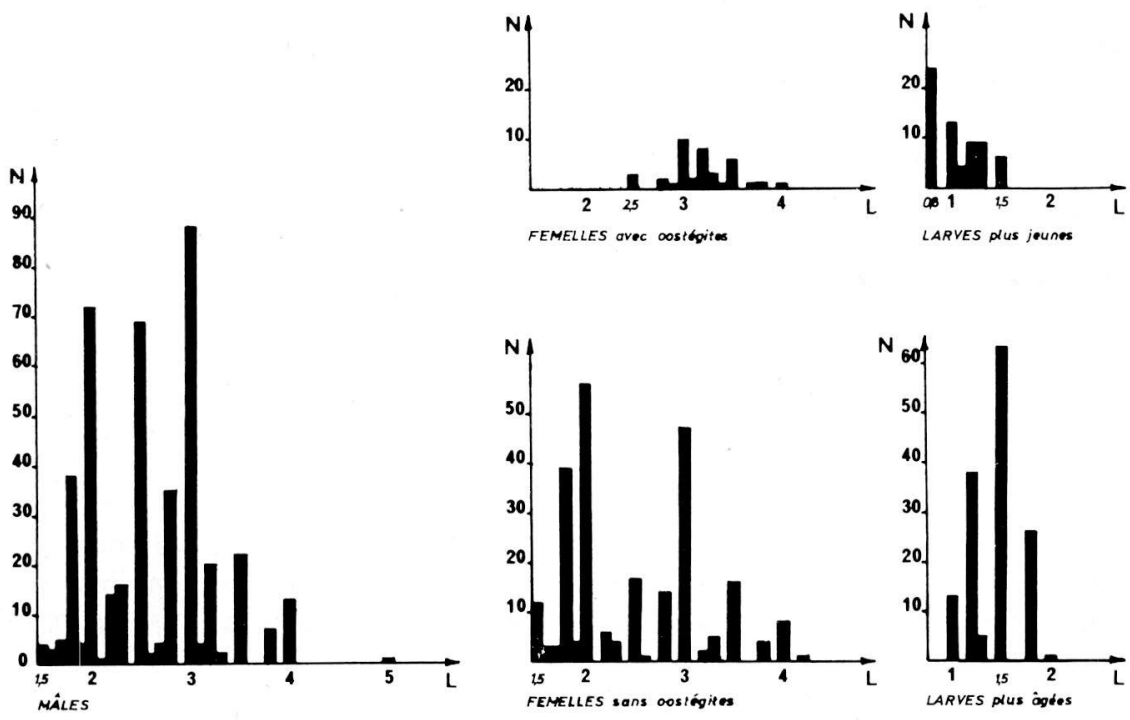

N nombre des animaux
$\mathrm{L}$ langueur du corps on mm

Fig. 2. Proasellus slavus ssp. n. Longueur du corps des mâles, femelles et larves.

Répartition des larves et des femelles avec marsupium d'après la période et le lieu de leur capture

Le programme des périodes de prise des échantillons a été limité par diverses conditions, et nous avons seulement des données sporadiques pour le printemps, l'été (début, milieu, fin) et la fin de l'hiver.

La profondeur jusqu'à laquelle nous avons pu faire des recherches dans le substrat a été limitée par la longueur de la sonde, c'est-à-dire par l'équipement nécessaire à son enfoncement. C'est pourquoi nous ne pouvons parler de la distribution de la population que jusqu'à une profondeur de $2 \mathrm{~m}$. Bien que lors de nos recherches nous ayons pu évaluer quelques autres facteurs écologiques abiotiques, nous n'envisagerons ici que la température de l'eau.

Nous avons récolté des embryons et des larves de 0,5 jusqu'à $2 \mathrm{~m}$ de profondeur, de même que des femelles avec oostégites fonctionnels. La zone qui est de toute évidence favorable à la reproduction s'étend assez loin sous le courant de 
la rivière (6 m sont prouvés), mais sous le rivage, c'est-à-dire sous le banc de sable, pas à plus de $1,5 \mathrm{~m}$, tout au moins pour la profondeur ayant fait l'objet des recherches.

Les données selon les saisons montrent que les échantillons du début et du milieu de l'été sont plus abondants (Mestrov et al., 1976) et que le nombre absolu de larves et d'embryons est plus grand en cette saison qu'au printemps, à la fin de l'été et en automne.

D'abondants échantillons provenant du début et du milieu de l'été, à une température de $18^{\circ} \mathrm{C}$ ou $19-20,3^{\circ} \mathrm{C}$ ont révèlé un nombre relativement grand d'individus en cours de développement; mais aucun de ceux pris à la fin de l'été et à la même température n'en contiennent et ils sont inférieurs numériquement.

$\mathrm{Au}$ printemps, nous avons un cas semblable, mais à une température sensiblement inférieure $\left(10,2-11,5^{\circ} \mathrm{C}\right)$ c'est-à-dire que les échantillons sont inférieurs quant au nombre, la densité de la population est petite, et en ce qui concerne les larves une seule a été capturée.

A la fin de l'hiver, la température de l'eau est encore inférieure $\left(8^{\circ} \mathrm{C}\right)$ et on a trouvé quelques larves jeunes et un embryon.

\section{DISCUSSION}

Le milieu hyporhéique de gravier et de sable de la Drave, en aval du confluent de la Mura, héberge une importante population de Crustacés Isopodes Proasellus slavus ssp. n. L'espèce à laquelle appartient cette forme était considérée comme "habitant des grands réservoirs d'eau souterraine" (Sket, 1963). L'holotype de cette sous-espèce qui n'a pas encore de nom, a été trouvé dans un puits. Cependant, dans la localité où ont eu lieu nos recherches il en existe, même malgré le degré de pollution relativement élevé, une population nombreuse dans les eaux interstitielles des alluvions. Elle s'y maintient et s'y renouvelle, ce qui témoigne que cette sous-espèce habite naturellement un tel biotope.

L'analyse de la population par sexe sur la base de nos récoltes a montré que les mâles prédominent. Le rapport du nombre des mâles par rapport au nombre des femelles de l'échantillon global est de 1,6:1. Mais la possibilité de changements temporaires des effectifs d'un sexe en faveur de l'autre n'est pas exclue, comme cela apparaît chez quelques espèces d'animaux.

Cette particularité de Proasellus slavus ssp. n. est tout à fait opposée à celle trouvée dans la population d'Asellides interstitiels endémiques, comme par exemple Stenasellus hungaricus thermalis, où le rapport permanent des sexes est 1:2 en faveur des femelles (Lattinger-Penko, 1970).

Un plus grand nombre d'échantillons aurait peut-être pu donner des résultats différents de ceux dont nous disposons actuellement pour Proasellus.

Les mesures de la łongueur du corps de quelques centaines d'exemplaires de chaque sexe classent cette sous-espèce parmi les plus petits Asellides et même parmi les plus petites sous-espèces de son espèce.

La longueur maximale du corps des mâles de cette population est de $5 \mathrm{~mm}$, et celle des femelles de $4,2 \mathrm{~mm}$; mais des femelles de $5 \mathrm{~mm}$ capturées par $\mathrm{F}$. Velkovrh à Gornja Radgona (Sket, 1963) sont également connues. 
Les plus petites femelles avec marsupium mesuraient $2,5 \mathrm{~mm}$ et les plus grandes $4 \mathrm{~mm}$, leur marsupium parfaitement développé délimitant un volume relativement grand. Sur la base des constatations faites sur le nombre d'oufs embryonnés dans le marsupium ( 15 dans une femelle de $3 \mathrm{~mm}$ ), il semble que la fertilité des Proasellus slavus ssp. n. soit analogue à celle des animaux cavernicoles Proasellus cavaticus, P. racovitzai et Asellus aquaticus cavernicolus. Chez le premier, les plus petites femelles ovigères $(3,4-4 \mathrm{~mm})$ ne donnent pas

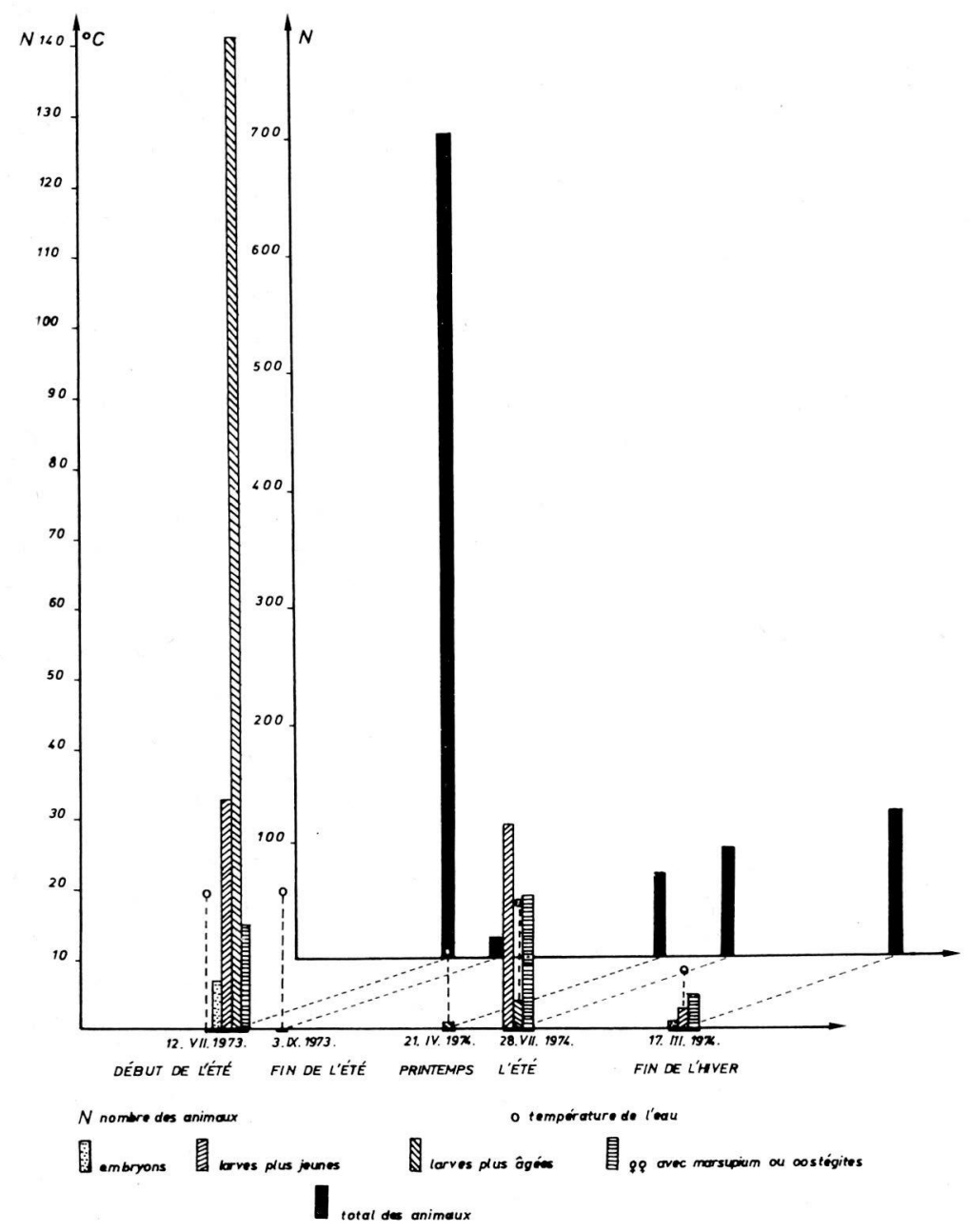

Fig. 3. Proasellus slavus ssp. n. Abondance et distribution saisonnière des larves et femelles avec la poche incubatrice en comparaison avec celles de la population complète. 
plus de 10 jeunes alors que les plus grandes $(5,5 \mathrm{~mm})$ en ont jusqu'à 46 (Henry, 1965). Chez le second, Proasellus racovitzai, la femelle de $6,4 \mathrm{~mm}$ portait 33 embryons (Henry et Magniez, 1972). Chez Asellus aquaticus cavernicolus, les femelles de 5-8 mm donnent jusqu'à 60 descendants (Lattinger-Penko, in lit.), vérifiant ainsi le fait général que l'effectif d'oeufs par portée est proportionnel à la taille de la femelle.

Parmi les exemplaires classés comme larves, nous avons pu discerner 3 types dont deux sont appelés larves plus jeunes et un larves plus âgées. Ces formes ne sont pas étudiées en détail et les noms sont provisoires, bien que les différences morphologiques entre elles soient significatives.

Les dimensions du corps des jeunes larves et des larves plus âgées dans les limites de 1 et 1,5 $\mathrm{mm}$ se recouvrent, à l'instar des Stenasellus hungaricus thermalis, ce qui signifie que la rapidité de la croissance en longueur de certains individus est en retard sur la vitesse du processus de métamorphose.

Bien que les données soient insuffisantes (les échantillons n'ont pas été prélevés tous les mois), elles indiquent que les Proasellus slavus ssp. n. se reproduisent pendant la plus grande partie de l'année et qu'il existe un maximum saisonnier, comme cela a été constaté par example chez Proasellus cavaticus (Henry, 1965) et Troglocaris anophtalmus (Juberthie-Jupeau, 1975). L'activité reproductrice accrue se manifeste par un effectif plus grand de jeunes en cours de développement et de femelles avec marsupium au début et au milieu de l'été (Fig. 3).

En cette saison, nous avons récolté, à une température moyenne de $19,5^{\circ} \mathrm{C}$, un grand nombre de larves et de femelles avec marsupium, alors qu'à la même température, il n'y en a plus à la fin de l'été. Par contre, à la fin de l'hiver, à une température bien inférieure $\left(8^{\circ} \mathrm{C}\right)$, nous avons de nouveau trouvé des larves. Il existe une possibilité qu'il faudrait vérifier au moyen d'expériences à savoir que de brusques changements de température pourraient être la cause d'un début d'activité sexuelle, comme chez le Gastéropode terrestre Radix peregra (Krkać, 1973).

La deuxième cause de l'intensité inégale de la reproduction est probablement due à la concentration différente des détritus servant de nourriture. On a remarqué sur le terrain que la quantité de matières organiques en décomposition varie dans le temps et l'espace et que les fortes densités de détritus et de population correspondent, ce qui a été corroboré par les travaux de Husmann.

Il reste encore cependant à faire des recherches plus fondamentales sur le terrain ainsi que des expériences avec l'espèce en cause. 


\section{CONCLUSIONS}

Ces premières données sur la biologie du Crustacé Isopode hypogé Proasellus slavus ssp. n. permettent de faire les constatations suivantes:

- cette sous-espèce habite le biotope interstitiel avec une population de composition complète.

- dans cette population, les mâles prédominent par leur nombre.

- la longueur maximale du corps des deux sexes est $5 \mathrm{~mm}$, par conséquent cette forme fait partie des Asellides les plus petits.

- les femelles portent dans leur marsupium un nombre d'oeufs ou de larves relativement grand, proportionnellement à celui de ce que nous appelons les cavernicoles récents tels que Proasellus cavaticus et Asellus aquaticus cavernicolus.

- il est vraisemblable que les animaux se multiplient durant toute l'année, mais que dans l'activité reproductrice il existe une périodicité saisonnière dont le maximum est au début de l'été.

\section{RÉSUMÉ}

L'analyse du peuplement du milieu hyporhéique de la Drave indique que le Proasellus slavus ssp. n. est l'un des plus petits Asellides 'connus et qu'il vit dans un biotope interstitiel en peuplements permanents dont la densité varie dans l'espace et dans le temps. Nos observations montrent que la reproduction a lieu toute l'année, mais elle présente un maximum au début de l'été. Les femelles malgré la petitesse de l'espèce peuvent donner un nombre relativement grand de descendants.

\section{BIBLIOGRAPHIE}

Henry, J-P., 1969: Remarques sur le cycle biologique d'Asellus cavaticus Leydig (Crustacé Isopode troglobie). Actes IVe CIS, 4-5, 1-408, Ljubljana.

Henry, J-P., Magniez, G., 1972: Observations sur un Aselle obscuricole de France: Proasellus racovitzai n. sp. (Crustacea Isopoda Asellota). Int. J. Speleol., 4, 171-188.

Juberthie-Jupeau, L., 1975: La ponte d'un Crustacé décapode souterrain: cycle saisonnier et influence biologique. Ann. Spéléol., 30, 1, 167-171.

Krkač, N., 1973: Promjena temperature zone - stimulans za odlaganje jaja vrste Radix peregra O. F. Müller. Ekologija, 8, 1, 131-138.

Lattinger-Penko, R., 1970: Analiza prirodne populacije Stenasellus hungaricus thermalis Meštrov

1960 (Crustacea, Isopoda). Ekologija, 5, 1, 117-124.
Data on the biology of an underground Crustacean, Asellus aquaticus cavernicolus Racovitza (Crustacea, Isopoda). Sous presse.

Meštrov, M., Lattinger-Penko, R., Tavčar, V.: La dynamique de la population d'isopode Proasellus slavus ssp. n. et les larves de Chronomides dans l'hyporheique de la Drava du point de vue de la pollution. Int. J. Speleol. 8, 1-2, 00-00.

Sktt, B., 1963: Asellus slavus Remy (Crust., Isopoda) v Jugoslaviji. Razprave SAZU, Class. IV, VII, 179-197. 